\title{
[Revision]
}

\section{Consensus Statement of the International Summit on Intellectual Disability and Dementia Related to Nomenclature ${ }^{1}$}

\author{
Janicki, M.P. ${ }^{1}$, McCallion. P. ${ }^{2}$, Splaine, M. ${ }^{3}$, Santos, F.H. ${ }^{4}$, Keller, S.M ${ }^{5}$, Watchman, K. ${ }^{6}$ \\ ${ }^{1}$ University of Illinois at Chicago, USA \\ ${ }^{2}$ University at Albany, New York USA \\ ${ }^{3}$ Splaine Consulting, Columbia, Maryland USA \\ ${ }^{4}$ São Paulo State University, Bauru, Brazil \\ ${ }^{5}$ American Academy of Developmental Medicine and Dentistry, Lumberton, New Jersey USA \\ ${ }^{6}$ University of Stirling, Scotland
}

Correspondence Address:

Matthew P. Janicki

University of Illinois at Chicago

Department of Disability and Human Development (MC626)

1640 W. Roosevelt Road

Chicago, IL 60608 USA

Email: mjanicki@uic.edu

Running head: Summit Statement on Nomenclature

Key words: Alzheimer's, dementia, intellectual disability, nomenclature, terminology

Please cite as: Janicki M, McCallion P, Splaine M, Santos FH, Keller S \& Watchman K (2017) Consensus Statement of the International Summit on Intellectual Disability and Dementia Related to Nomenclature, Intellectual and Developmental Disabilities, 55 (5), pp. 338-346.https://doi.org/10.1352/1934-9556-55.5.338

\footnotetext{
${ }^{1}$ The authors are the primary members of the Summit working group on nomenclature. This consensus statement was developed as an output from the 2016 International Summit on Intellectual Disability and Dementia, held in Glasgow, Scotland, 13-14 October 2016, and hosted by the University of Stirling and University of the West of Scotland, funded by the RS MacDonald Trust, the Scottish Government, and Alzheimer Scotland. Collaborating sponsors included the National Task Group on Intellectual Disabilities and Dementia Practices (NTG) in the United States and the University of Illinois at Chicago. The Summit was composed of individuals and representatives of many international and national organizations with a stake in issues related to adults with intellectual disability affected by dementia. The contents of this statement were partially developed under a grant from the United States Department of Health and Human Services, Administration for Community Living (ACL), National Institute on Disability, Independent Living, and Rehabilitation Research (NIDILRR) Grant \# 90RT5020-03-00. However, those contents do not necessarily represent the policy of the US Department of Health and Human Services, nor the endorsement by the US federal government. The opinions expressed represent those of the Summit participants and of the NTG.
} 


\begin{abstract}
The 2016 International Summit on Intellectual Disability and Dementia examined the terminology used to define and report on dementia in publications related to intellectual disability (ID) and found mixed uses of terms associated with dementia or causative diseases. It noted that language related to dementia in the ID field often lacks precision and could lead to a misunderstanding of the condition(s) under discussion. Most articles related to ID and dementia reporting clinical or medical research generally provide a structured definition of dementia or related terms; social care articles tend toward term use without definition. Toward terminology standardization within studies/reports on dementia and ID, the Summit recommended that a consistent approach is taken that ensures (a) growing familiarity with dementia-related diagnostic, condition-specific, and social care terms (as identified in the working group's report), (b) creating a guidance document on accurately defining and presenting information about individuals or groups referenced, and (c) that in reports on neuropathologies or cognitive decline or impairment, definitions are used and data include subjects' ages, sex, level of ID, residential situation, basis for dementia diagnosis, presence of Down syndrome (or other risk conditions), years from diagnosis, and if available, scores on objective measures of changing function.
\end{abstract}




\section{Introduction}

Language can and does shape and form our perceptions of a condition or situation. As noted by Alzheimer's Europe (2013, p. 11), "Some words and metaphors are used liberally and paint a very biased picture of dementia, whilst others are avoided and considered demeaning, depersonalizing, and insulting. Even standard medical terms are sometimes used with great caution due to an awareness of the possible impact on people's lives and wellbeing. Words clearly matter. They describe, communicate and reinforce our current perceptions of dementia."

Nomenclature (or terminology) is a system of names or terms, or the rules for forming these terms, in a discipline or field of study. Reviews of publications (i.e., journal articles, book chapters, reports, documents, and plans) involving intellectual disability show varied uses of terms associated with dementia or the diseases or conditions causing it (Janicki et al., 2016). Terms in use (including dementia, Alzheimer's, and other similar descriptors) often lack precision or consistency and may be used loosely and applied inappropriately. As with publications in the mainstream dementia field, this misuse may be attributed to a lack of understanding of the distinction in the terms, the nuances involved with neuropathologies, language usage preferences and translational difficulties, or inconsistent use of language, as well as absence of or inconsistency in an agreed upon core group of methods used in diagnosis. Notably, language related to dementia in the intellectual disability field often lacks precision in relation to its peculiarities from onset to progression and often leads to a misunderstanding or incomplete representation of the condition(s) under discussion.

A lack of precision in general applications, as witnessed by recent efforts in the mainstream Alzheimer's and dementia field to address the same issue, appears common. This concern over coherency regarding terminology was discussed by Taylor (2016) at the Alzheimer's Disease-Related Dementias 2016 Summit, held in the United States under the auspice of the National Institutes of Health (Foundation for the National Institutes of Health, 2016). Taylor listed a range of terms that are in use in the field and stimulated a dialogue on dementia-related nomenclature regardless of the etiology, specific clinical syndromes, or underlying etiologies. She noted a number of problems stemming from inconsistency in terminology, such as Alzheimer's disease being used synonymously with dementia, low public 
awareness of other forms of dementia, and a lack of recognition that 'Alzheimer's disease' services are also applicable for those persons affected by non-Alzheimer's based dementias.

The problems stemming from inconsistent terminology were also addressed at meeting of the U.S. National Advisory Council on Alzheimer's Research, Care, and Services, which under the National Alzheimer's Project Act (NAPA) is responsible for the U.S. National Plan to Address Alzheimer's Disease (Department of Health and Human Services, 2012). Among the issues raised was the rationale for the adoption of updated clinical terminology within the most recent Diagnostic and Statistical Manual of Mental Disorders (DSM-5; APA, 2013), and the range of terms in use for various dementias, leading to a desire to introduce terminological coherency in planning documents (Petersen, 2016).

The updated clinical terminology refers to the DSM-5's replacement of the terms 'mild cognitive impairment' and 'dementia' with 'minor neurocognitive disorder' and 'major neurocognitive disorder', recognizing distinctions between distinct or progressive stages. The APA's DSM-5 changes were designed to recognize a spectrum perspective and varying incidence of locus of decline related to different etiologies, and provide more clinical and diagnostic precision in identifying variations in the presentation of dementia, particularly when dementia was attributed to causes other than Alzheimer's disease and presenting without the classic symptoms inherent in Alzheimer's (Simpson, 2014). It also was an attempt to remove some of the stigma associated with the word 'dementia'. Within the new terminology, the focus is on decline rather than deficit in function and the term expands the categories to include other etiological conditions (e.g., HIV and acquired brain injuries), which do not necessarily occur in association with aging. Concerns about standardization of language have been increased by this change, as the new terms have clinical utility but not general societal usage, and have not yet generally been adopted by the major Alzheimer's or other dementia-related organizations internationally in public education and fund raising campaigns. Introducing the term 'neurocognitive' is also potentially confusing when intellectual disability also has aspects of neurocognitive dysfunction, and support for the use of the word 'disorder' is questioned in the intellectual disability field as it sees the term as stigmatizing (Weymeyer, 2013). Another consideration is that while the new DSM-5 is in use in some Western countries, the use of the definitions and diagnostic coding found in the ICD-10 (WHO, 1992) is more prevalent 
worldwide, leading to some disparities in what terms clinicians and authors may use in medical and research reports, respectively.

Consequently, the National Advisory Council inserted a recommendation into the 2016 U.S. National Plan Update that stated that "Emphasis should be given to the standardization of terminology in dealing with cognitive and dementing disorders." Further, to address this issue head-on, the 2016 Update recommended that "an integrated conference should be convened to develop consistent language for cognitive disorders among scientists, care providers and the public... [and there is a need to] engage all of the stakeholders around these issues to reach a consensus for the benefit of persons with dementia, their family members and caregivers, and the scientific and service communities" (ASPE, 2016).

\section{Conveyance of the notion of dementia}

Language usage and terminology is germane to how people affected by dementia are viewed to the extent terms used minimize stigma (Garand, Lingler, Conner, \& Dew, 2010). Clearly, 'people-first' language [e.g., adults with dementia] creates more positive imagery and can go far to minimize negative perceptions. Language uses such as 'adults with dementia' are preferable to terms such as 'demented, victim, and sufferer', which tend to be pejorative and maximize stigmatization (DEEP, 2015). Such positive terms also focus on the individuals rather than the condition.

Communicating the definition and process of dementia to people with intellectual disability creates a further conundrum. To enable understanding, language and phrasing needs to be at a word-level that organizations communicating with the general public, caregivers, advocates, or adults affected by dementia, can effectively convey the concepts associated with dementia. Further, the concepts need to be communicated in a manner so as to explain the essence and implications, and convey the seriousness of the condition while not creating confusion or undue anxiety. Siberski (2012) has covered the concern of how the general public integrates clinical conceptualizations and reacts to information about diagnoses.

An aspect of communicating is the ease with which the general population grasps the concept behind a clinical disease or neuropathology - so avoiding 'high science language' in documents directed toward general readership audiences can facilitate understanding. Using 
terms and definitions that are in common usage can help to ensure the communication of messages. Conversely, creating definitions that minimize the impact of the course and eventuality of brain changes leading to dementia can leave conditions untreated (Siberski, 2012). To examine the success of concept conveyance to the general public through the level of language used, the Summit working group analyzed both the reading ease and grade level of definitions conveyed by different dementia, health, and disability organizations when defining dementia (see Janicki et al., 2016 for the full report listing the terms and definitions). Of the 16 definitions extracted from major dementia-related organization websites and documents, the reading ease level ranged from 60.70 to $0.0(X=26.68)$ and the grade level ranged from 7.8 to $18.6(\mathrm{X}=13.84){ }^{2}$ It appears that most of the definitions were expressed using complex concepts, even when directed at the general public. For those directed toward professionals, despite clarity that dementia is not a disease itself but a group of behavioral and/or function symptoms that can accompany certain diseases or conditions, the definitions used were more in the realm of technical language.

Language and terms are a further challenge for intellectual disability organizations, as they have to convey both information about the condition and forewarn caregivers of the condition's severity and eventual impact. How wording is constructed depends on the audience and message to be conveyed, and often the explanations lean toward complexity. This is true even among Down syndrome advocacy organizations, where the definitions and explanations of dementia (and Alzheimer's disease) used to inform their constituents tend toward complexity see Table 1. However, when materials are developed specifically for people with intellectual disability, concepts have been explained in a simpler manner. As an example of a plain language definition of dementia, the Down's Syndrome Scotland's (n.d.) publication for adults with intellectual disability, states "Dementia is an illness in the brain. It affects many things, but mostly the way people remember and do things."

\section{Insert Table 1 About Here}

While it was not the function of this Summit statement to offer a standardized definition of 'dementia', it was recognized that the condition's primary features are characterized by the

\footnotetext{
${ }^{2}$ In general, the higher the reading ease score, the more generally understandable wording is; with respect to the grade level, lower scores correspond with lower grade level reading abilities.
} 
progressive loss of brain function that occurs with certain neuropathological diseases or trauma, often associated, but not necessarily related, to aging (others have addressed this characterization in depth - see Chertkow et al., 2013; Small et al., 1997). Such neuropathologies include, among others, Alzheimer's disease, Parkinson's disease, Pick's disease, and cerebrovascular accidents. The neuropathologies vary and have differing effects - but all have in common the eventual expression of progressive behavioral and cognitive changes and decline generally defined as dementia. This expression, in most instances, includes eventual marked memory disorders and impaired reasoning, personality and behavioral changes, and loss of self-care, mobility, and communication. The principal aspects of the expression are related to the type of dementia (i.e., based on the etiology). However, it is recognized that features that may initially be taken for dementia-like behavioral dysfunctions may instead be the result of adverse drug reactions, depression, psychological trauma, or a range of other causes - with their defining feature a clearing or reduction of symptoms upon treatment (Desai \& Grossberg, 2001).

Further, it was not the intent of this Summit statement to rectify inconsistencies in dementia-associated language usage across the general field of dementia (see Janicki et al., 2016, for examples of the variations), but to promote the understanding of the distinctions among the terms in prevalent usage, and advance the application of precise usage and agreement on dementia-related terms that are commonly used in association with intellectual disability. Such a result would advance clearer differentiation and appropriate usage of clinical terms such as: dementia, Alzheimer's disease, cognitive impairment, Alzheimer's disease spectrum, pre-clinical Alzheimer's disease, dementia due to Alzheimer's disease, mild cognitive impairment due to Alzheimer's disease, early versus late onset disease, and mild, moderate and advanced dementia (Alzheimer's disease), and others. We are not proposing a standard definition of dementia or acceptance of a range of associated terms that would be applied in work in intellectual disability - no such consensus on term usage exists in the general dementia literature or policy documents (save for those noted earlier in the DSM-5 (APA, 2013) or ICD-10 (WHO, 1992) for diagnostic purposes). We are suggesting that workers/researchers/educators do provide an operational definition of dementia of their choosing so as to offer readers a clear understanding of the variables and notions related to the population being described.

\section{Applications of terms}


How terms are used can affect clarity in understanding which subject population is being referenced and what conditions are being discussed. In a sampling of available articles and reports related to intellectual disability and dementia undertaken by the Summit's working group, term-usage was found to be variable and at times less than clear (see Janicki et al., 2016). Authors in general used 'dementia' as a common term defining the nature of the condition, there was often mixed reference to Alzheimer's disease, dementia of the Alzheimer's type, and generic 'dementia,' without linkage to standard definitions (such as those in the DSM-5 and ICD-10). There was also an absence of defining whether the dementia being reported was progressive. While the usage served the intended purpose - of defining the general concern of the article - it left open interpretations of how the condition was identified in the subjects, to what degree it was progressing (or if progression was a concern), and what may have been the underlying neuropathology. Some of these data may not always be readily available in social care research, but should be available in medical/health related research and in articles advancing or relying upon standardized assessments.

Defining the subject population also has import and implications for research related to dementia in the intellectual disability field. The Summit working group's study also noted the high preponderance of articles in the intellectual disability and dementia literature that focused predominantly on Down syndrome (Janicki et al., 2016). Given the high risk for Alzheimer's disease among adults with Down syndrome this is not unexpected. However, it is important that authors are clear when reporting results and generalizing effects that the information provided relates to dementia in adults with Down syndrome (if this was the subject population), as there may be differential patterns of onset, effect, and mortality in the greater population of adults with intellectual disability who do not have Down syndrome. Often in articles and reports the number or percentage of subjects with Down syndrome (and their sex and ages) is not identified within the study population; this may confound generalizability unto the greater population of adults with intellectual disability, and misinform on the epidemiology of dementia. Also, as most studies involving adults with Down syndrome relate to dementia of the Alzheimer's type, this also should be noted in the subject descriptions, and/or any variations in forms of dementia should be noted. Such detail will also highlight the number of study participants where an accurate diagnosis has not been made and the type of dementia is not known. 
As what is popularly considered chronological aging can span 30 or more years, dementia-oriented studies should consider grouping data into age categories that reflect the younger-older and older-older grouping of subjects. Given that early-onset dementia is prevalent in adults with Down syndrome, the age span for younger-older may begin in the 40s and the older-older with age 60. When adults absent Down syndrome are the focus of studies then younger-older may begin in the 60s, and later age groupings may include those aged 75 and older - similar to general population reports. Such groupings can help establish patterns in incidence, related features, such as co-morbidities, and staging. Also, when reporting subject recruitment, methodology sections in reports should contain concise definitions of dementia and at what dementia stage recruitment occurred. Although, identifying stage of dementia can be confounded by the degree and nature of the intellectual disability, efforts should still be made to define it as accurately as possible (Krinsky-McHale \& Silverman, 2013). When reporting staging, terminology should reflect the spectrum of cognitive impairment when that is known in the study subjects.

The Summit working group observed that most articles related to intellectual disability and dementia were using prevalent dementia-related terms, rather than the DSM-5 terminology (e.g., neurocognitive disorders); this is also observed in the general dementia literature. Also, that the articles reporting assessment and clinical or medical research were more apt to provide a definition of dementia or related terms, while social care articles tended to only use the term itself and not define it or provide substantiation of study subject diagnoses. The working group suggested that it would increase research and practice recommendation utility if social care articles also provided definitions and the basis for ascribing dementia to the persons in the studies, thus offering more precision and permitting cross-article comparisons and contrasts. Further, as many of the articles examined failed to define the subject population with respect to age, sex, level of intellectual disability, etiology, and other demographic variables, the standardization of reporting subject information recommended here would also increase utility. Also, judicious use of common terms among key words in manuscript submissions will aid in locating relevant articles following publication (e.g., Alzheimer's disease, cognitive impairment, dementia, Down syndrome, neurocognitive disorders, neuropathology, intellectual disability, etc.) 


\section{Commentary}

The intent of this Summit statement is to reflect discussion at the international meeting in Scotland to promote the understanding of the distinctions among terms in prevalent use with respect to intellectual disability and dementia, and to advance usage that more accurately reflects the concepts cited, and agreement on commonly used dementia and intellectual disability-related terms. Terminology needs precision to accurately convey the conditions and services being discussed. The Summit proposes that authors, irrespective of the focus of the article, provide operational definitions of conditions being considered, ancillary demographic and clinical data, study population parameters, particularly the numbers (and percentages) of subjects with Down syndrome, and information about how diagnoses were obtained, as well as the protocols and instruments employed. In social care articles, authors should offer a definition or explanation of the program(s) or service(s) under discussion. Readers would then be offered a clearer understanding of the population being described and the features being studied, and thus replication in future studies and comparisons across existing studies would be facilitated.

\section{Recommendations}

The Summit goals were to address issues associated with nomenclature (or terminology), seek agreement on a common taxonomy related to cognitive impairment and dementia among persons with intellectual disability and encourage use of standardized terminology within studies and reports on dementia and intellectual disability. With this in mind, the Summit recommends:

- To promote positive imagery, organizations, researchers, educators and workers adopt and use image enhancing language when describing persons with intellectual disability affected by dementia and avoid language that stigmatizes or dehumanizes;

- To promote standardized understanding of the meaning of terms used to describe services and conditions related to dementia and intellectual disability, that a taxonomy be adopted for general use. A taxonomy of unvetted terms related to defining dementia and dementia services prevalent in dementia and intellectual disability publications is found in the appendix of the full Summit report (see Janicki et al., 2016) and can offer user guidance on the development of a formal taxonomy; 
- To help with reporting and describing events or conditions related to intellectual disability and dementia, that a guidance document be created on accurately defining and presenting information about individuals or the group being referenced to include both formal diagnostic criteria and general definitions of various dementias. We recommend that the guidance document be supported by a consortium of associated intellectual disability, gerontology, and dementia groups, and appear in a relevant publication (for archival purposes); and

- To standardize reporting of subjects and outcomes from studies, researchers, educators and workers follow recommendations for harmonizing data in reports addressing neuropathologies, behavioral and functional changes, cognitive decline or impairment. Such reports should use recommended definitions and at a minimum include the subjects' ages, sex, level of intellectual disability, residential situation, co-morbidities, basis for dementia diagnosis, presence of Down syndrome (or other risk condition), years from diagnosis, and if available, scores on an objective measure of changing function from a recognized and validated dementia scale.

\section{References}

Alzheimer Europe. (2013). The ethical issues linked to the perceptions and portrayal of dementia and people with dementia. Luxembourg, Luxembourg: Author. Accessed from: http://www.alzheimer-europe.org/Ethics/Ethical-issues-in-practice/The-ethical-issueslinked-to-the-perceptions-and-portrayal-of-dementia-and-people-with-dementia

ASPE. (2016). National Plan to Address Alzheimer's Disease: 2016 Update. Washington, DC: Author. Accessed from: https://aspe.hhs.gov/report/national-plan-address-alzheimersdisease-2016-update

American Psychiatric Association. (2013). Diagnostic and statistical manual of mental disorders (5th ed.). Washington, DC: Author. 
Chertkow, H., Feldman, H.H., Jacova, C., \& Massoud, F. (2013). Definitions of dementia and predementia states in Alzheimer's disease and vascular cognitive impairment: consensus from the Canadian conference on diagnosis of dementia. Alzheimer's Research \& Therapy, 5(Suppl 1), S2. DOI: 10.1186/alzrt198.

Dementia Engagement \& Empowerment Project. (2015). Dementia words matter: Guidelines on language about dementia. (DEEP Guidelines). Accessed from: http://dementiavoices.org.uk/wp-content/uploads/2015/03/DEEP-Guide-Language.pdf

Desai, A.K. \& Grossberg, G.T. (2001). Recognition and management of behavioral disturbances in dementia. Primary Care Companion Journal of Clinical Psychiatry, 3(3), 93-109.

Department of Health and Human Services. (2012). National plan to address Alzheimer's disease. Washington, DC: Author.

Down's Syndrome Scotland. (n.d.). What is dementia? A booklet about dementia for adults who have a learning disability. Edinburgh, Scotland: Author. Accessed from: http://aadmd.org/sites/default/files/whatisdementiabooklet.pdf

Garland, L., Lingler, J.H., Connor, K.O., \& Dew, M.A. (2010). Diagnostic labels, stigma, and participation in research related to dementia and mild cognitive impairment. Research in Gerontological Nursing, 2(2), 112-121.

Janicki, M.P., McCallion, P., Splaine, M., Santos, F.H., Keller, S., \& Watchman, K. (2016). Report of the summit working group on nomenclature and dementia \& intellectual disability: Topic report from the International Summit on Intellectual Disability and Dementia. Glasgow, Scotland, October 13-14, 2016. http://www.learningdisabilityanddementia.org/id-dementia-summit.html 
Krinsky-McHale, S.J., \& Silverman, W. (2013). Dementia and mild cognitive impairment in adults with intellectual disability: issues of diagnosis. Developmental Disabilities Research Reviews, 18(1), 31-42.

Petersen, R.C. (2016). Terminology heterogeneity. Presentation at the April 29, 2016 meeting of the Advisory Council on Alzheimer's Research, Care, and Services, Washington, DC. Accessed from: https://aspe.hhs.gov/advisory-council-april-2016-meeting-presentationterminology-heterogeneity

Simpson, J.R. (2014). DSM-5 and neurocognitive disorders. Journal of the American Academy of Psychiatry and the Law, 42(2), 159-164.

Siberski, J. (2012). Dementia and DSM-5: Changes, cost, and confusion. Aging Well, 5(6), 12. Accessed from: http://www.todaysgeriatricmedicine.com/archive/110612p12.shtml

Small, G.W., Rabins, P.V., Barry, P.P., Buckholtz, N.S., DeKosky, S.T., Ferris, S.H., ... Tune, L.E. (1997). Diagnosis and treatment of Alzheimer disease and related disorders: consensus statement of the American Association for Geriatric Psychiatry, the Alzheimer's Association, and the American Geriatrics Society. JAMA, The Journal of the American Medical Association, 278(16), 1363-1371.

Taylor, A. (2010, March). Dementia nomenclature. Presentation at the Alzheimer's DiseaseRelated Dementias 2016 Summit. Bethesda, Maryland (USA), March 29-30, 2016.

World Health Organization. (1992). The ICD-10 classification of mental and behavioural disorders: Clinical descriptions and diagnostic guidelines. Geneva: Author.

Wehmeyer, M.L. (2013). Disability, disorder, and identity. Intellectual and Developmental Disabilities, 51(2), 122-126. doi: 10.1352/1934-9556-51.2.122 


\begin{tabular}{|c|c|c|}
\hline Source & Website & Statement \\
\hline $\begin{array}{l}\text { Down Syndrome } \\
\text { International } \\
\text { (INT) }\end{array}$ & $\begin{array}{l}\text { https://ds-int.org/resource/adults-18- } \\
\text { ageing }\end{array}$ & $\begin{array}{l}\text { "Dementia is a frequently voiced concern about people with Down syndrome. There is a } \\
\text { tendency for people with Down syndrome to develop dementia at a younger age than in } \\
\text { the general population. However, research indicates that the overall percentage of adults } \\
\text { with Down syndrome who develop dementia is similar to that of the population who do } \\
\text { not have Down syndrome. It is not inevitable that those with Down syndrome will } \\
\text { develop dementia." }\end{array}$ \\
\hline $\begin{array}{l}\text { Down Syndrome } \\
\text { Research } \\
\text { Foundation } \\
\text { (Canada) }\end{array}$ & $\begin{array}{l}\text { http://www.dsrf.org/information/down- } \\
\text { syndrome-and-alzheimer-s/ }\end{array}$ & $\begin{array}{l}\text { "People with Down syndrome (DS) are at an increased risk of Alzheimer's disease (AD) } \\
\text { because of the presence of three copies of chromosome } 21 . \text { Chromosome } 21 \text { contains } \\
\text { over } 300 \text { genes including several that are associated with AD; the amyloid precursor } \\
\text { protein gene (APP) is one of these genes. This and other genes are associated with the } \\
\text { plaques that accumulate in the brain as well as inflammation and oxidative stress that } \\
\text { takes place early in development and progressively leads to mitochondrial impairment, } \\
\text { degeneration of neuronal pathways, accelerated aging and loss of brain cells in the } \\
\text { cerebral cortex and hippocampus. Although in DS AD brain pathology is frequently seen in } \\
\text { the } 40 \text { 's not everyone with DS exhibits the clinical signs of Alzheimer's, which usually } \\
\text { happens in the } 50 \text { 's and } 60 \text { 's, even though all have the brain pathology." }\end{array}$ \\
\hline $\begin{array}{l}\text { Down's } \\
\text { Syndrome } \\
\text { Association (UK) }\end{array}$ & $\begin{array}{l}\text { http://www.downs-syndrome.org.uk/for- } \\
\text { families-and-carers/health-and-well- } \\
\text { being/getting-older/alzheimers-disease/ }\end{array}$ & $\begin{array}{l}\text { "Alzheimer's disease is a type of dementia that gradually destroys brain cells, affecting a } \\
\text { person's memory and their ability to learn, make judgments, communicate and carry out } \\
\text { basic daily activities. Alzheimer's disease is characterized by a gradual decline that } \\
\text { generally progresses through three stages: early, middle and late stage disease. } * * * \\
\text { Dementia is not a disease in its own right. Dementia is an umbrella term covering more } \\
\text { than } 100 \text { different conditions associated with a cognitive decline. One of these conditions } \\
\text { is Alzheimer's disease." }\end{array}$ \\
\hline $\begin{array}{l}\text { National Down } \\
\text { Syndrome } \\
\text { Society (USA) }\end{array}$ & $\begin{array}{l}\text { http://www.ndss.org/Resources/Aging- } \\
\text { Matters/Alzheimers-Disease/An- } \\
\text { Introduction-to-Alzheimers-Disease/ }\end{array}$ & $\begin{array}{l}\text { "Alzheimer's disease is a type of dementia that gradually destroys brain cells, affecting a } \\
\text { person's memory and their ability to learn, make judgments, communicate and carry out } \\
\text { basic daily activities. Alzheimer's disease is characterized by a gradual decline that } \\
\text { generally progresses through three stages: early, middle and late stage disease. These } \\
\text { three stages are distinguished by their general features, which tend to progress gradually } \\
\text { throughout the course of the disease. Alzheimer's disease is not inevitable in people with } \\
\text { Down syndrome. While all people with Down syndrome are at risk, many adults with } \\
\text { Down syndrome will not manifest the changes of Alzheimer's disease in their lifetime. } \\
\text { Although risk increases with each decade of life, at no point does it come close to } \\
\text { reaching } 100 \%{ }^{* * *} \text { Estimates show that Alzheimer's disease affects about } 30 \% \text { of people } \\
\text { with Down syndrome in their } 50 \text { s. By their } 60 \text { s, this number comes closer to } 50 \% . "\end{array}$ \\
\hline
\end{tabular}




\begin{tabular}{|l|l|l|}
\hline $\begin{array}{l}\text { Down Syndrome } \\
\text { Australia (AUS) }\end{array}$ & $\begin{array}{l}\text { http://www.downsyndrome.org.au/docu } \\
\text { ments/Ageing_Resource_Health_Check.p } \\
\text { df }\end{array}$ & $\begin{array}{l}\text { "Alzheimer's disease is a progressive degenerative condition of the brain that results in a } \\
\text { gradual change, over years, in a person's ability to think, remember and perform tasks of } \\
\text { daily living. In ways that scientists don't yet understand, the extra copies of genes present } \\
\text { in people with Down syndrome cause developmental problems and health issues. } \\
\text { Scientists think that the increased risk of dementia in individuals with Down syndrome } \\
\text { may also result from the extra gene present on chromosome 21. Some studies suggest } \\
\text { that } 30 \text { per cent of people with Down syndrome aged in their 50's have Alzheimer's } \\
\text { disease and more than 75 per cent aged } 65 \text { and older have Alzheimer's disease." }\end{array}$ \\
& & \multicolumn{2}{|c}{} \\
\end{tabular}

